\title{
Mie Scattering by a Conducting Sphere Coated Uniaxial Single-Negative Medium
}

\author{
You-Lin Geng \\ Institute of Antenna and Microwaves, Hangzhou Dianzi University, Xiasha, Hangzhou, Zhejiang 310018, China \\ Correspondence should be addressed to You-Lin Geng, gengyoulin@yahoo.com.cn
}

Received 24 June 2012; Accepted 4 November 2012

Academic Editor: James R. Kelly

Copyright () 2012 You-Lin Geng. This is an open access article distributed under the Creative Commons Attribution License, which permits unrestricted use, distribution, and reproduction in any medium, provided the original work is properly cited.

We propose an accurate analytical method to compute the electromagnetic scattering from three-dimensional (3D) conducting sphere coated uniaxial anisotropic single-negative (SNG) medium. Based on the spherical vector wave functions (SVWFs) in uniaxial anisotropic medium, the electromagnetic field in homogeneous uniaxial SNG medium and free space can be expressed by the SVWFs in uniaxial SNG medium and free space. The continued boundary conditions of electromagnetic fields between the uniaxial SNG medium and free space are applied, and the tangential electrical field is vanished in the surface of conducting sphere, the coefficients of scattering fields in free space can be derived, and then the character of scattering of conducting sphere coated homogeneous uniaxial SNG medium can be obtained. Some numericals are given in the end.

\section{Introduction}

Recently, the development of metamaterials, which was first introduced by Veselago in 1968 [1], has attracted more and more attention in the physical and engineering communities. Metamaterials, which are a kind of new artificial media, have been studied intensively since the concept of perfect lenses was proposed by Pendry in 2000 [2] and the negative index of refraction was experimentally verified by Smith et al. in 2001 [3]. Another breakthrough in metamaterials happened in 2006, when a method for controlling electromagnetic (EM) fields was proposed using inhomogeneous and anisotropic metamaterials [4] and the reduced invisible cloak was successfully realized using metamaterials at microwave frequencies [5]. Since then, more and more researchers have investigated the properties and applications of anisotropic metamaterials.

The EM scattering problem for penetrable objects is very important to study material properties and arbitrary dielectric lenses. There are many classical methods for analysing traditional two-dimensional (2D) and three-dimensional (3D) dielectric scattering problems [6-11]. In recent years, there have also been many theoretical investigations on the study of EM characteristics for double-negative (DNG) media [12-15], where both the permittivity and permeability are negative.

However, "single-negative" (SNG) materials in which only one of the material parameters, not both, has a negative real value may also possess interesting properties when they are paired in a conjugate manner. These media include the epsilon-negative (ENG) media, in which the real part of permittivity is negative, but the real permeability is positive, and the mu-negative (MNG) media, in which the real part of permeability is negative, but the real permittivity is positive. For instance, the idea of constructing an effective metamaterials by having layers of SNG media has been explored by Fredkin and Ron in [16], and some works have been done by Alù and Engheta [17-19]. It is well known that the negative medium is not isotropic; it is anisotropy; some works have been done between acoustic or electromagnetic waves and anisotropic metamaterials [2022 ], and this paper discusses the scattering by a conducting sphere coated uniaxial anisotropic SNG medium.

Based on the spherical vector wave functions in uniaxial sphere [11] and uniaxial sphere of left-handed material [13], electromagnetic fields in homogeneous uniaxial anisotropic single-negative medium can be expressed as the addition of the first and second SVWFs in uniaxial SNG medium. 


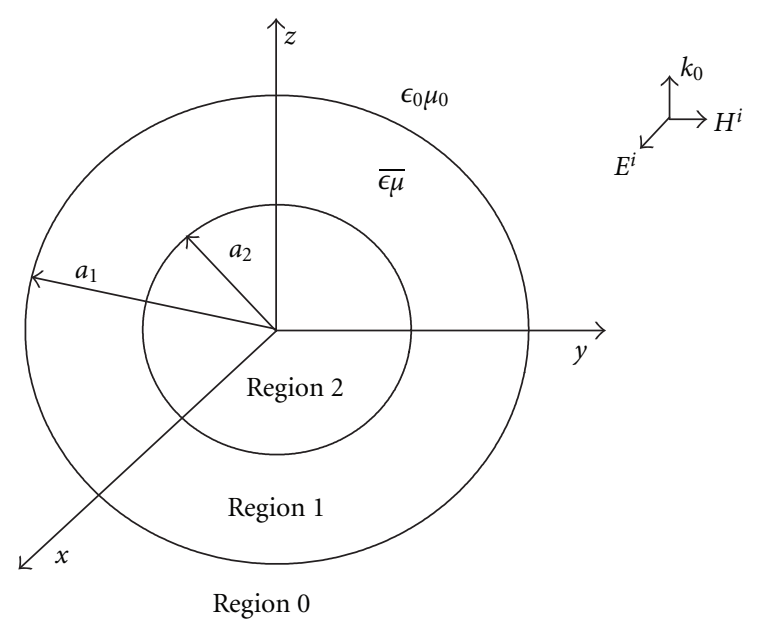

Figure 1: Geometry of a plane wave scattering by a plasma anisotropic spherical shell.

Applying the continued boundary conditions of electromagnetic fields, and the tangential electric field vanishing in the interface of the conducting sphere, the scattering coefficients of scattering fields in free space can be derived. The numerical results between the present method in this paper and Mie theory of scattering by a conducting sphere coated isotropic SNG medium are given, a good agreement is obtained as we expected, and some new numerical results are given in this paper.

In the subsequent analysis, an $\exp (-i \omega t)$ time dependence is assumed for electromagnetic field quantities but suppressed throughout the treatment.

\section{Formulas}

Let us consider a conducting sphere coated homogeneous uniaxial SNG medium illuminated by an incident plane wave. As illuminated in Figure 1, the coated sphere with outer radius $a_{1}$ and inner radius $a_{2}$ is located at the coordinate origin. On the surface of the inner conducting sphere, the uniaxial SNG medium with permittivity tensor $(\bar{\epsilon})$ and permeability tensor $(\bar{\mu})$ is coated with thickness $d(=$ $\left.a_{1}-a_{2}\right)$. It is assumed that the incident wave propagates in the $+\hat{z}$ direction, the incident electric field has unity of amplitude, and is polarized in the $+\hat{x}$ direction.

The electric field vector wave equation in such a sourcefree uniaxial anisotropic SNG medium can be written in the following form $[6,11]$ :

$$
\nabla \times\left[\overline{\boldsymbol{\mu}}^{-1} \cdot \nabla \times \mathbf{E}(\mathbf{r})\right]-\omega^{2} \overline{\boldsymbol{\epsilon}} \cdot \mathbf{E}(\mathbf{r})=0,
$$

where $\mathbf{E}$ denotes the electric field, while $\overline{\boldsymbol{\epsilon}}$ and $\bar{\mu}$ represent the permittivity tensor and the permeability tensor of uniaxial SNG medium; the expression are $[11,13]$

$$
\overline{\boldsymbol{\epsilon}}=\left[\begin{array}{ccc}
\epsilon_{t} & 0 & 0 \\
0 & \epsilon_{t} & 0 \\
0 & 0 & \epsilon_{z}
\end{array}\right], \quad \boldsymbol{\mu}=\left[\begin{array}{ccc}
\mu_{t} & 0 & 0 \\
0 & \mu_{t} & 0 \\
0 & 0 & \mu_{z}
\end{array}\right] .
$$

Some works were done about this negative medium in the past years $[21,22]$, and in this paper, either of the permittivity or permeability is negative; that is, the permittivity $\overline{\boldsymbol{\epsilon}}$ is negative while the permeability $\bar{\mu}$ is positive, or on the contrary, the permeability $\bar{\mu}$ is negative while the permittivity $\overline{\boldsymbol{\epsilon}}$ is positive. Using Fourier transform $[6,11]$, the expansion of plane wave factors in terms of spherical vector wave functions in isotropic medium [23], and the properties of spherical Bessel functions [24], the electromagnetic fields in the uniaxial anisotropic SNG medium can be obtained as follows:

$$
\begin{gathered}
\mathbf{E}=\sum_{l=1}^{2} \sum_{q=1}^{2} \sum_{m n n^{\prime}} F_{m n^{\prime} q}^{(l)} \int_{0}^{\pi}\left[A_{m n q}^{e}\left(\theta_{k}\right) \mathbf{M}_{m n}^{(l)}\left(\mathbf{r}, k_{q}\right)\right. \\
+B_{m n q}^{e}\left(\theta_{k}\right) \mathbf{N}_{m n}^{(l)}\left(\mathbf{r}, k_{q}\right) \\
\left.+C_{m n q}^{e}\left(\theta_{k}\right) \mathbf{L}_{m n}^{(l)}\left(\mathbf{r}, k_{q}\right)\right] \\
\times P_{n^{\prime}}^{m}\left(\cos \theta_{k}\right) k_{q}^{2} \sin \theta_{k} d \theta_{k}, \\
\mathbf{H}=\sum_{l=1}^{2} \sum_{q=1}^{2} \sum_{m n n^{\prime}} F_{m n^{\prime} q}^{(l)} \int_{0}^{\pi}\left[A_{m n q}^{h}\left(\theta_{k}\right) \mathbf{M}_{m n}^{(l)}\left(\mathbf{r}, k_{q}\right)\right. \\
\quad+B_{m n q}^{h}\left(\theta_{k}\right) \mathbf{N}_{m n}^{(l)}\left(\mathbf{r}, k_{q}\right) \\
\left.\quad+C_{m n q}^{h}\left(\theta_{k}\right) \mathbf{L}_{m n}^{(l)}\left(\mathbf{r}, k_{q}\right)\right] \\
\times P_{n^{\prime}}^{m}\left(\cos \theta_{k}\right) k_{q}^{2} \sin \theta_{k} d \theta_{k},
\end{gathered}
$$

where $n^{\prime}$ and $n$ are summed up both from 0 to $+\infty$ while $m$ is summed up from $-n$ to $n$, and $\mathbf{r}$ is pointing to the $(\theta, \phi)$ direction in the spherical coordinates. The coefficients, $F_{m n q}^{(l)}$, are unknown, as in $[11,13] . A_{m n q}^{p}\left(\theta_{k}\right), B_{m n q}^{p}\left(\theta_{k}\right), C_{m n q}^{p}\left(\theta_{k}\right)$ (where $p=e$ or $h$ ), and $k_{q}$ are functions of $\theta_{k}$ and they have been derived in $[11,13]$. The vector wave functions, $\mathbf{M}_{m n}^{(l)}$, $\mathbf{N}_{m n}^{(l)}$, and $\mathbf{L}_{m n}^{(l)}$ are spherical vector wave functions and they are also shown in $[11,23]$.

The incident electromagnetic fields (designated by the superscript inc) can be expanded in an infinite series in isotropic spherical vector wave functions $[11,23]$ :

$$
\begin{aligned}
\mathbf{E}^{\mathrm{inc}}= & \sum_{m n}\left[a_{m n}^{x} \mathbf{M}_{m n}^{(1)}\left(\mathbf{r}, k_{0}\right)+b_{m n}^{x} \mathbf{N}_{m n}^{(1)}\left(\mathbf{r}, k_{0}\right)\right] \\
& \times\left[\delta_{m, 1}+\delta_{m,-1}\right], \\
\mathbf{H}^{\mathrm{inc}}= & \frac{k_{0}}{i \omega \mu_{0}} \sum_{m n}\left[a_{m n}^{x} \mathbf{N}_{m n}^{(1)}\left(\mathbf{r}, k_{0}\right)+b_{m n}^{x} \mathbf{M}_{m n}^{(1)}\left(\mathbf{r}, k_{0}\right)\right] \\
& \times\left[\delta_{m, 1}+\delta_{m,-1}\right],
\end{aligned}
$$


where the expansion coefficients are defined as:

$$
\begin{gathered}
a_{m n}^{x}= \begin{cases}i^{n+1} \frac{2 n+1}{2 n(n+1)}, & m=1, \\
i^{n+1} \frac{2 n+1}{2}, & m=-1 ;\end{cases} \\
b_{m n}^{x}= \begin{cases}i^{n+1} \frac{2 n+1}{2 n(n+1)}, & m=1, \\
-i^{n+1} \frac{2 n+1}{2}, & m=-1 ;\end{cases} \\
\delta_{s, l}= \begin{cases}1, & s=l, \\
0, & s \neq l .\end{cases}
\end{gathered}
$$

According to the radiation condition of an outgoing wave (attenuating to zero at infinity) and the asymptotic behavior of spherical Bessel functions, only $h_{n}^{(1)}$ should be retained in the radial functions; therefore, the expansion of scattered fields (designated by the superscript $s$ ) are

$$
\begin{array}{r}
\mathbf{E}^{s}=\sum_{m n}\left[A_{m n}^{s} \mathbf{M}_{m n}^{(3)}\left(\mathbf{r}, k_{0}\right)+B_{m n}^{s} \mathbf{N}_{m n}^{(3)}\left(\mathbf{r}, k_{0}\right)\right], \\
\mathbf{H}^{s}=\frac{k_{0}}{i \omega \mu_{0}} \sum_{m n}\left[A_{m n}^{s} \mathbf{N}_{m n}^{(3)}\left(\mathbf{r}, k_{0}\right)+B_{m n}^{s} \mathbf{M}_{m n}^{(3)}\left(\mathbf{r}, k_{0}\right)\right],
\end{array}
$$

where the coefficients, $A_{m n}^{s}$ and $B_{m n}^{s}$ ( $n$ varies from 0 to $+\infty$ while $m$ changes from $-n$ to $n$ ), are unknowns to be determined, $\mathbf{M}_{m n}^{(l)}\left(\mathbf{r}, k_{0}\right)$ and $\mathbf{N}_{m n}^{(l)}\left(\mathbf{r}, k_{0}\right)$ denote the spherical vector wave functions defined in $[11,23]$, and $k_{0}=\omega\left(\epsilon_{0} \mu_{0}\right)^{1 / 2}$ identifies the wave number of free space, respectively.

Applying the boundary conditions at the surface of uniaxial anisotropic SNG medium, for example, when $r=$ $a_{2}$, the expansion coefficients of electromagnetic fields in uniaxial anisotropic medium can be obtained by the following equations:

$$
\begin{gathered}
\sum_{l=1}^{2} \sum_{q=1}^{2} \sum_{n^{\prime}=0}^{\infty} F_{m n^{\prime} q}^{(l)} \int_{0}^{\pi} A_{m n q}^{e} z_{n}^{(l)}\left(k_{q} a_{2}\right) \\
\times P_{n^{\prime}}^{m}\left(\cos \theta_{k}\right) k_{q}^{2} \sin \theta_{k} d \theta_{k}=0, \\
\sum_{l=1}^{2} \sum_{q=1}^{2} \sum_{n^{\prime}=0}^{\infty} F_{m n^{\prime} q}^{(l)} \int_{0}^{\pi}\left\{B_{m n q}^{e} \frac{1}{k_{q} r} \frac{d}{d r}\left(r z_{n}^{(l)}\left(k_{q} r\right)\right)\right. \\
\left.\times C_{m n q}^{e} \frac{z_{n}^{(l)}\left(k_{q} r\right)}{r}\right\}_{r=a_{2}} \\
\times P_{n^{\prime}}^{m}\left(\cos \theta_{k}\right) k_{q}^{2} \sin \theta_{k} d \theta_{k}=0
\end{gathered}
$$

and $r=a_{1}$ and can be obtained the following expression:

$$
\begin{gathered}
\sum_{l=1}^{2} \sum_{q=1}^{2} \sum_{n^{\prime}=0}^{\infty} F_{m n^{\prime} q}^{(l)} \int_{0}^{\pi} Q_{m n q}^{(l)} P_{n^{\prime}}^{m}\left(\cos \theta_{k}\right) k_{q}^{2} \sin \theta_{k} d \theta_{k} \\
=\left[\delta_{m, 1}+\delta_{m,-1}\right] a_{m n}^{x} \frac{i}{\left(k_{0} a_{1}\right)^{2}}, \\
\sum_{l=1}^{2} \sum_{q=1}^{2} \sum_{n^{\prime}=0}^{\infty} F_{m n^{\prime} q}^{(l)} \int_{0}^{\pi} R_{m n q}^{(l)} P_{n^{\prime}}^{m}\left(\cos \theta_{k}\right) k_{q}^{2} \sin \theta_{k} d \theta_{k} \\
=\left[\delta_{m, 1}+\delta_{m,-1}\right] b_{m n}^{x} \frac{i}{\left(k_{0} a_{1}\right)^{2}},
\end{gathered}
$$

where expansion coefficients $a_{m n}^{x}$ and $b_{m n}^{x}$ can be expressed in (7) and (8), and $z_{n}^{(l)}$ (where $l=1,2,3$, and 4) denotes an appropriate kind of spherical Bessel functions, $j_{n}, y_{n}$, $h_{n}^{(1)}$, and $h_{n}^{(2)}$, respectively. $Q_{m n q}^{(l)}$ and $R_{m n q}^{(l)}$ have the following expression:

$$
\begin{aligned}
Q_{m n q}^{(l)}= & \left\{A_{m n q}^{e} \frac{1}{k_{0} r} \frac{d}{d r}\left(r h_{n}^{(1)}\left(k_{0} r\right)\right) z_{n}^{(l)}\left(k_{q} r\right)\right. \\
& -\frac{i \omega \mu_{0}}{k_{0}}\left[B_{m n q}^{h} \frac{1}{k_{q} r} \frac{d}{d r}\left(r z_{n}^{(l)}\left(k_{q} r\right)\right)\right. \\
& \left.\left.+C_{m n q}^{h} \frac{z_{n}^{(l)}\left(k_{q} r\right)}{r}\right] \cdot h_{n}^{(1)}\left(k_{0} r\right)\right\}_{r=a_{1}} \\
R_{m n q}^{(l)}=\left\{\frac{i \omega \mu_{0}}{k_{0}} A_{m n q}^{h} \frac{1}{k_{0} r} \frac{d}{d r}\left(r h_{n}^{(1)}\left(k_{0} r\right)\right) z_{n}^{(l)}\left(k_{q} r\right)\right. & \\
& -\left[B_{m n q}^{e} \frac{1}{k_{q} r} \frac{d}{d r}\left(r z_{n}^{(l)}\left(k_{q} r\right)\right)+C_{m n q}^{e} \frac{z_{n}^{(l)}\left(k_{q} r\right)}{r}\right] \\
& \left.\cdot h_{n}^{(1)}\left(k_{0} r\right)\right\}_{r=a_{1}} .
\end{aligned}
$$

The integral in (12) to (15) can be easily calculated by Gauss integral [24], and from (12) to (15), it shows that

(i) firstly, the unknown coefficients $\left(F_{m n^{\prime} q}^{(l)}\right)$ of electromagnetic fields in the uniaxial anisotropic SNG medium can be obtained;

(ii) secondly, the coefficients $\left(A_{m n}^{s}, B_{m n}^{s}\right)$ of scattered fields in region 0 (free space) are calculated; and

(iii) lastly, the far scattering field of electromagnetic fields from a conductor sphere coated uniaxial anisotropic SNG medium by a plane wave and the radar crosssection are thus obtained.

\section{Numerical Results and Discussion}

In the last section, we have presented the necessary theoretical formulation of the electromagnetic fields of a plane wave scattered by a conducting sphere coated uniaxial anisotropic 


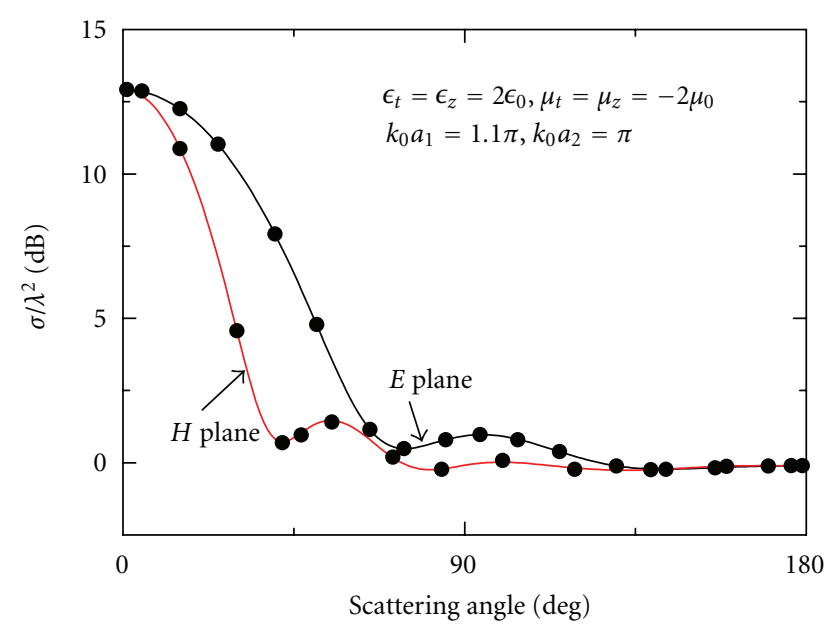

Figure 2: Radar cross sections (RCSs) versus scattering angle $\theta$ (in degrees): results of this paper (solid curve) and of Mie theory (black round).

SNG medium. To gain more physics insight into the problem, we will provide in this section some numerical solutions to the problem of electromagnetic scattering by a conducting sphere coated uniaxial anisotropic SNG medium.

Numerical computations have been performed by applying the theoretical formulae derived earlier in the previous sections. Since there are no numerical results of scattering by a conducting sphere coated uniaxial anisotropic SNG medium, in order to check the accuracy of the newly obtained numerical results, we performed one trial; that is, we calculated the radar cross-sections using the present method and the Mie theory (scattering by a conducting sphere coated homogeneous isotropic SNG medium). The results are shown in Figure 2, where electric dimensions of outer and inner spherical surfaces are $k_{0} a_{1}=1.1 \pi$ and $k_{0} a_{2}=$ $\pi$, while the permittivity and permeability tensor elements are $\epsilon_{t}=\epsilon_{z}=2 \epsilon_{0}, \mu_{t}=\mu_{z}=-2 \mu_{0}$, respectively, (where and subsequently, $\epsilon_{0}$ and $\mu_{0}$ stand for the free space permittivity and permeability, resp.).

From Figure 2, it is seen apparently that the radar cross sections calculated by using the two methods (i.e., the present method in this paper and Mie theory) are in very good agreement in both the $E$ - and $H$-planes, where the maximum number of $n^{\prime}$ used in (12) to (15) is only 6 to achieve the convergence. It partially verifies the correctness and applicability of our theory as well as the Fortran program codes.

After this, we obtain some new results unavailable elsewhere in the literature. Three examples are considered herein, and their radar cross sections are plotted in Figures 3,4 , and 5 .

Figure 3 represents radar cross sections of a conducting sphere coated uniaxial anisotropic SNG medium of more general uniaxial medium, where the permittivity and permeability tensor elements are characterized by $\epsilon_{t}=-2 \epsilon_{0}$, $\epsilon_{z}=-3 \epsilon_{0}$, and $\mu_{t}=3 \mu_{0}, \mu_{z}=2 \mu_{0}$, and the electric size of the uniaxial anisotropic SNG spherical shell is chosen as

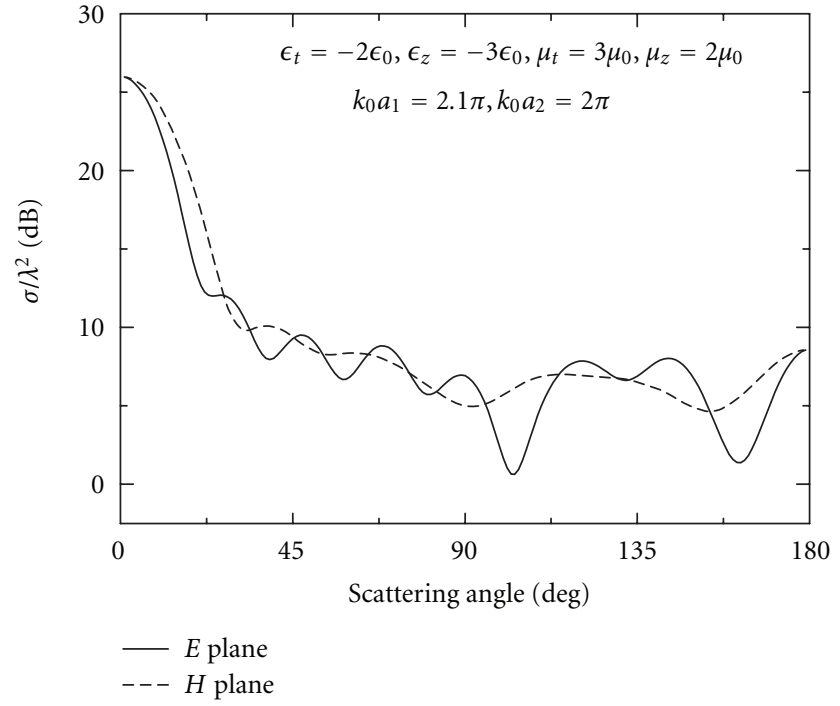

FIGURE 3: Radar cross-sections (RCSs) versus scattering angle $\theta$ (in degrees) in the $E$-plane (solid curve) and in the $H$-plane (shortdashed curve).

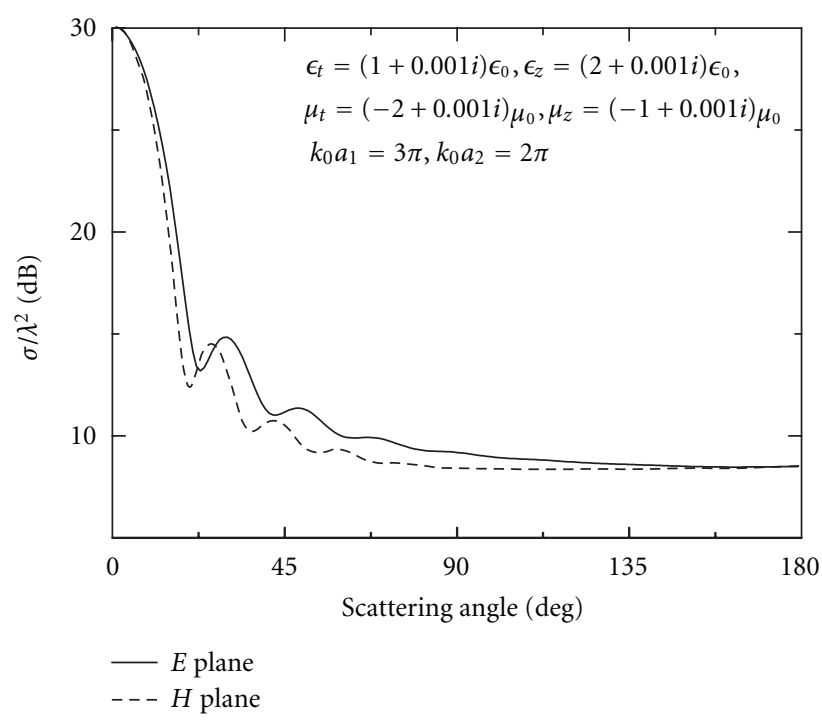

Figure 4: Radar cross sections (RCSs) versus scattering angle $\theta$ (in degrees) in the $E$-plane (solid curve) and in the $H$-plane (shortdashed curve).

$k_{0} a_{1}=2.1 \pi$ and $k_{0} a_{2}=2 \pi$. The maximum number $n^{\prime}$ in (12) to (15) to achieve a good convergence is found to be 16 .

Figure 4 gives a numerical result of scattering by a conducting sphere coated loss uniaxial SNG medium. The electric size of the uniaxial anisotropic SNG spherical shell is chosen as $k_{0} a_{1}=3 \pi$ and $k_{0} a_{2}=2 \pi$ under the illumination by an incident plane wave. The permittivity and permeability tensor parameters used for this case are $\epsilon_{t}=(1+0.001 i) \epsilon_{0}$, $\epsilon_{z}=(2+0.001 i) \epsilon_{0}$, and $\mu_{t}=(-2+0.001 i) \mu_{0}, \mu_{z}=$ $(-1+0.001 i) \mu_{0}$. As the electric dimension of the sphere is increased, the maximum number of $n^{\prime}$ used in (12) to 


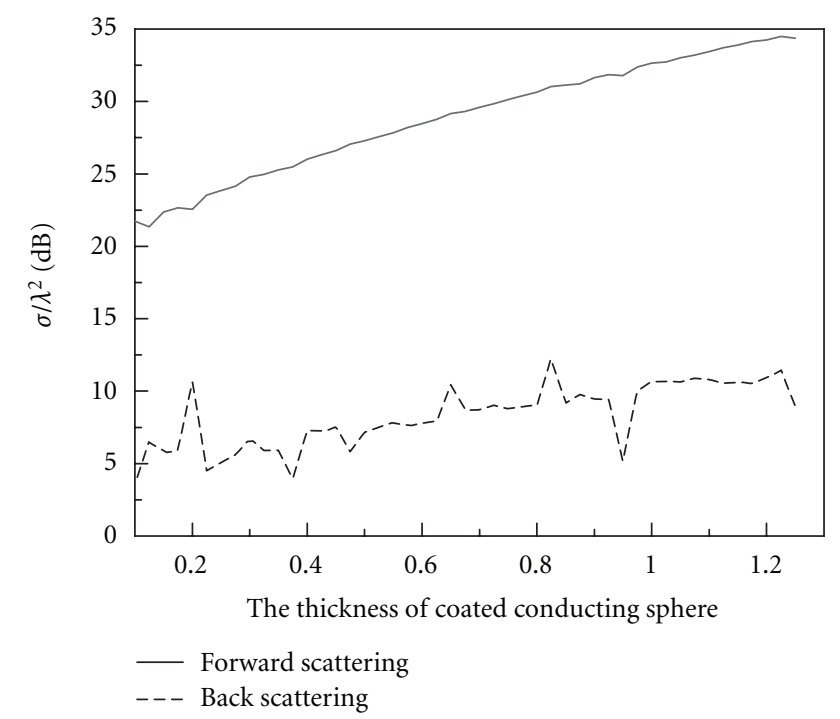

Figure 5: Radar cross sections (RCSs) versus the thickness of the uniaxial SNG medium in the forward (solid curve) and in the back (dashed curve) directions, when inner radius is fixed at $a_{2}=0.75 \lambda$. The parameters of the uniaxial SNG coating of the conducting sphere are assumed to be $\epsilon_{t}=-1.5 \epsilon_{0}, \epsilon_{t}=-3 \epsilon_{0}, \mu_{t}=3 \mu_{0}$, and $\mu_{z}=1.5 \mu_{0}$, respectively.

(15) must be significantly increased to 22 to achieve the convergence.

Later in this section, monostatic RCSs are given; for example, the forward RCS (the scattering angle $\theta=0$ ) and the back RCS (the scattering angle $\theta=\pi$ ) are given in Figure 5. The parameters of the coated uniaxial SNG medium are $\epsilon_{t}=-1.5 \epsilon_{0}, \epsilon_{z}=-3 \epsilon_{0}$, and $\mu_{t}=3 \mu_{0}, \mu_{z}=$ $1.5 \mu_{0}$, and the electric size of conducting sphere is $k_{0} a_{2}=$ $1.5 \pi$, and the change of coated uniaxial SNG medium is from $0.1 \lambda$ to $1.25 \lambda$ ( $\lambda$ is the wavelength of incident wave in free space) at the interval of 0.025 . From this Figure, the forward RCS of the core-shell system almost increases as the thickness of the uniaxial SNG coating increases. However, it is interesting to note that the back scattering will be oscillating. The RCS in the forward direction is always larger than that in the back direction.

\section{Conclusions}

The spherical vector wave function expansion solution to the plane wave scattering by a conducting sphere coated uniaxial anisotropic SNG medium is obtained analytically in this paper. The solution has only one-dimensional integral which can be calculated by Gauss integral [24] easily. Numerical results are obtained using the present method and compared with Mie theory and a fairly good agreement is observed. It is shown that the obtained solution is stable even for almost isotropic scatterers, since the proposed solution is an analytical one of a conducting sphere coated uniaxial SNG medium, and the result of the Mie theory is a special case of the present method. The general numerical results, including the conducting sphere coated lossy uniaxial SNG medium and resonance region, are given and are found reducible to those of spacial cases. The present analysis is believed to be useful in antenna and wave propagation.

\section{Acknowledgments}

This work is partially supported by Grant no. 60971047 of the National Natural Science Foundation of China (NSFC) and Grant no. Y1080730 of the Natural Science Foundation of Zhejiang Province of China.

\section{References}

[1] V. G. Veselago, "The electrodynamics of substances with simul-taneously negative values of $\varepsilon$ and $\mu$," Soviet Physics Uspekhi, vol. 10, no. 4, pp. 509-514, 1968.

[2] J. B. Pendry, "Negative refraction makes a perfect lens," Physical Review Letters, vol. 85, no. 18, pp. 3966-3969, 2000.

[3] D. R. Smith, R. A. Shelby, and S. Schultz, "Experimental verification of a negative index of refraction," Science, vol. 292, no. 5514, pp. 77-79, 2001.

[4] J. B. Pendry, D. Schurig, and D. R. Smith, "Controlling electromagnetic fields," Science, vol. 312, no. 5781, pp. 17801782, 2006.

[5] D. Schurig, J. J. Mock, B. J. Justice et al., "Metamaterial electromagnetic cloak at microwave frequencies," Science, vol. 314, no. 5801, pp. 977-980, 2006.

[6] X. B. Wu and K. Yasumoto, "Three-dimensional scattering by an infinite homogeneous anisotropic cylinder: an analytical solution," Journal of Applied Physics, vol. 82, no. 1, pp. 19962003, 1997.

[7] X. Q. Sheng and Z. Peng, "Analysis of scattering by large objects with off-diagobally annisotropic material using finite element-boundary integral-multilevel fast multipole algorithm," IET Microwaves, Antennas and Propagation, vol. 4, pp. 492-500, 2010.

[8] X. Q. Sheng and C. Q. Deng, "A simple and efficient implementation of the well-conditioned electric-field integral equation," IEEE Transactionson Antennas and Propagation, vol. 57, no. 2, pp. 582-586, 2009.

[9] T. J. Cui, W. C. Chew, A. A. Aydiner, and Y. H. Zhang, "Fastforward solvers for the low-frequency detection of buried dielectric objects," IEEE Transactions on Geoscience and Remote Sensing, vol. 41, no. 9, pp. 2026-2036, 2003.

[10] R. S. Chen and E. K. N. Yung, "An efficient method to analyze the $\mathrm{H}$-plane waveguide junction circulator with a ferrite sphere," IEEE Transactions on Microwave Theory and Techniques, vol. 49, no. 5, pp. 928-937, 2001.

[11] Y. L. Geng, X. B. Wu, L. W. Li, and B. R. Guan, "Mie scattering by a uniaxial anisotropic sphere," Physical Review E, vol. 70, no. 5, Article ID 056609, 2004.

[12] H. F. Ma, J. F. Zhang, X. Chen, Q. Cheng, and T. J. Cui, "CG-FFT algorithm for three-dimensional inhomogeneous and biaxial metamaterials," Waves in Random and Complex Media, vol. 19, no. 1, pp. 49-64, 2009.

[13] Y. L. Geng and S. L. He, "Analytical solution for electromagnetic scattering from a sphere of uniaxial left-handed material," Journal of Zhejiang University, Science A, vol. 7, no. 1, pp. 99-104, 2006.

[14] C. W. Qiu, H. Y. Yao, L. W. Li, S. Zouhdi, and T. S. Yeo, "Backward waves in magnetoelectrically chiral media: 
propagation, impedance, and negative refraction," Physical Review B, vol. 75, no. 15, Article ID 155120, 2007.

[15] M. Y. Wang, D. B. Ge, J. Xu, and J. Wu, "FDTD study on back scattering of conducting sphere coated with doublenegatibe metamaterials," International Journal of Infrared and Millimeter Waves, vol. 28, no. 2, pp. 199-206, 2007.

[16] D. R. Fredkin and A. Ron, "Effective left-handed (negative index) composite material," Applied Physics Letters, vol. 81, no. 10, pp. 1753-1755, 2002.

[17] A. Alù and N. Engheta, "Achieving transparency with plasmonic and metamaterial coatings," Physical Review E, vol. 72, no. 1, Article ID 016623, 2005.

[18] A. Alù and N. Engheta, "Guided modes in a waveguide filled with a pair of single-negative (SNG), double-negative (DNG), and/or double-positive (DPS) layers," IEEE Transactions on Microwave Theory and Techniques, vol. 52, no. 1, pp. 199-210, 2004.

[19] A. Alù and N. Engheta, "Polarizabilities and effective parameters for collections of spherical nanoparticles formed by pairs of concentric double-negative, single-negative, and/or doublepositive metamaterial layers," Journal of Applied Physics, vol. 97, no. 6, Article ID 094310, 2005.

[20] J. Christensen and F. J. G. de Abajo, "Anisotropic metamaterials for full control of acoustic waves," Physical Review Letters, vol. 108, no. 12, Article ID 124301, 2012.

[21] D. R. Smith, P. Rye, D. C. Vier, A. F. Starr, J. J. Mock, and T. Perram, "Design and measurement of anisotropic metamaterials that exhibit negative refraction," IEICE Transactions on Electronics, vol. 87, no. 3, pp. 359-370, 2004.

[22] L. B. Hu and Z. F. Lin, "Imaging properties of uniaxially anisotropic negative refractive index materials," Physics Letters, vol. 313, no. 4, pp. 316-324, 2003.

[23] D. Sarkar and N. J. Halas, "General vector basis function solution of Maxwell's equations," Physical Review E, vol. 56, no. 1, pp. 1102-1112, 1997.

[24] M. Abramowitz and I. A. Stegun, Handbook of Mathematical Functions With Formulas, Graphs, and Mathematical Tables, Dover Publications, New York, NY, USA, 1972. 

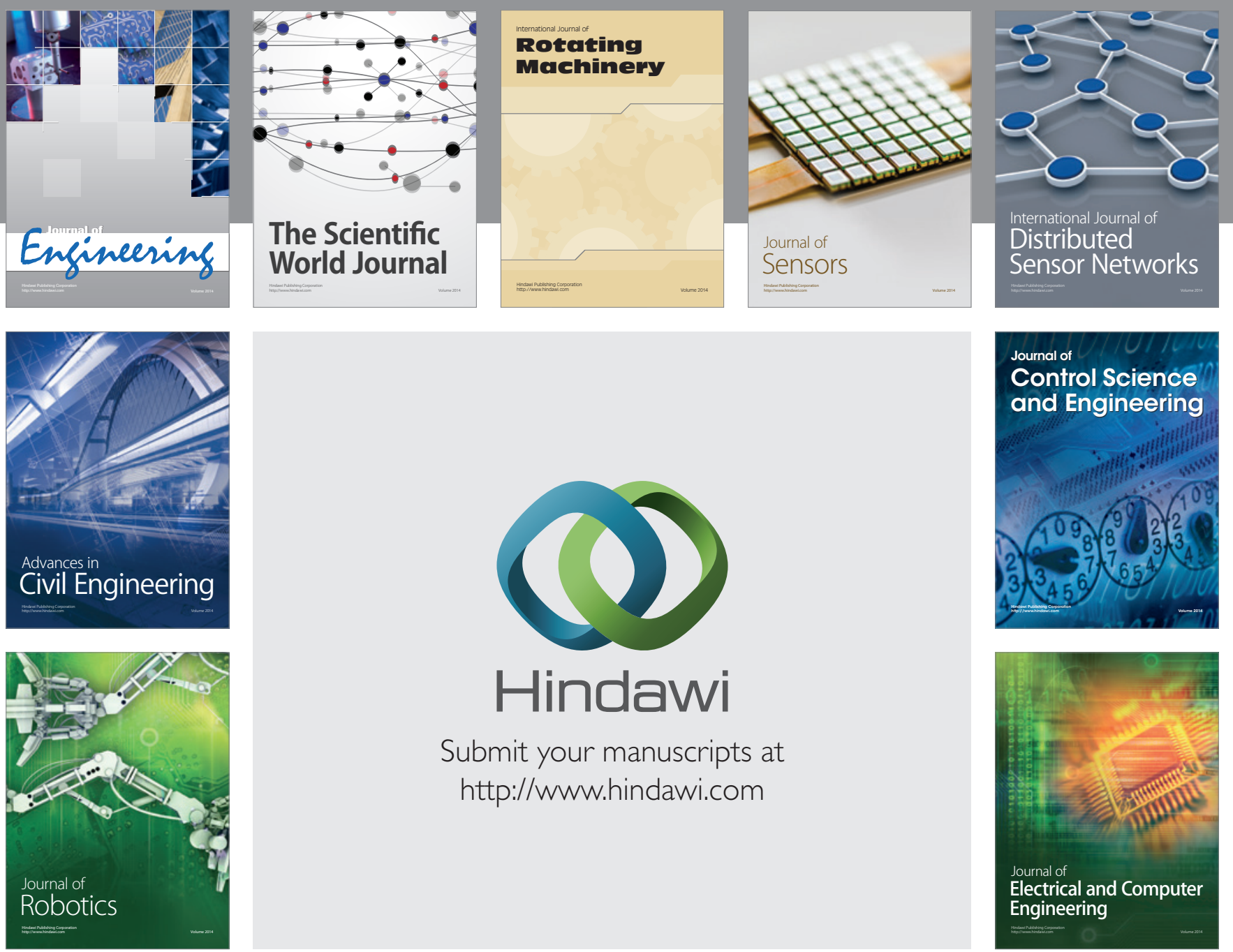

Submit your manuscripts at

http://www.hindawi.com
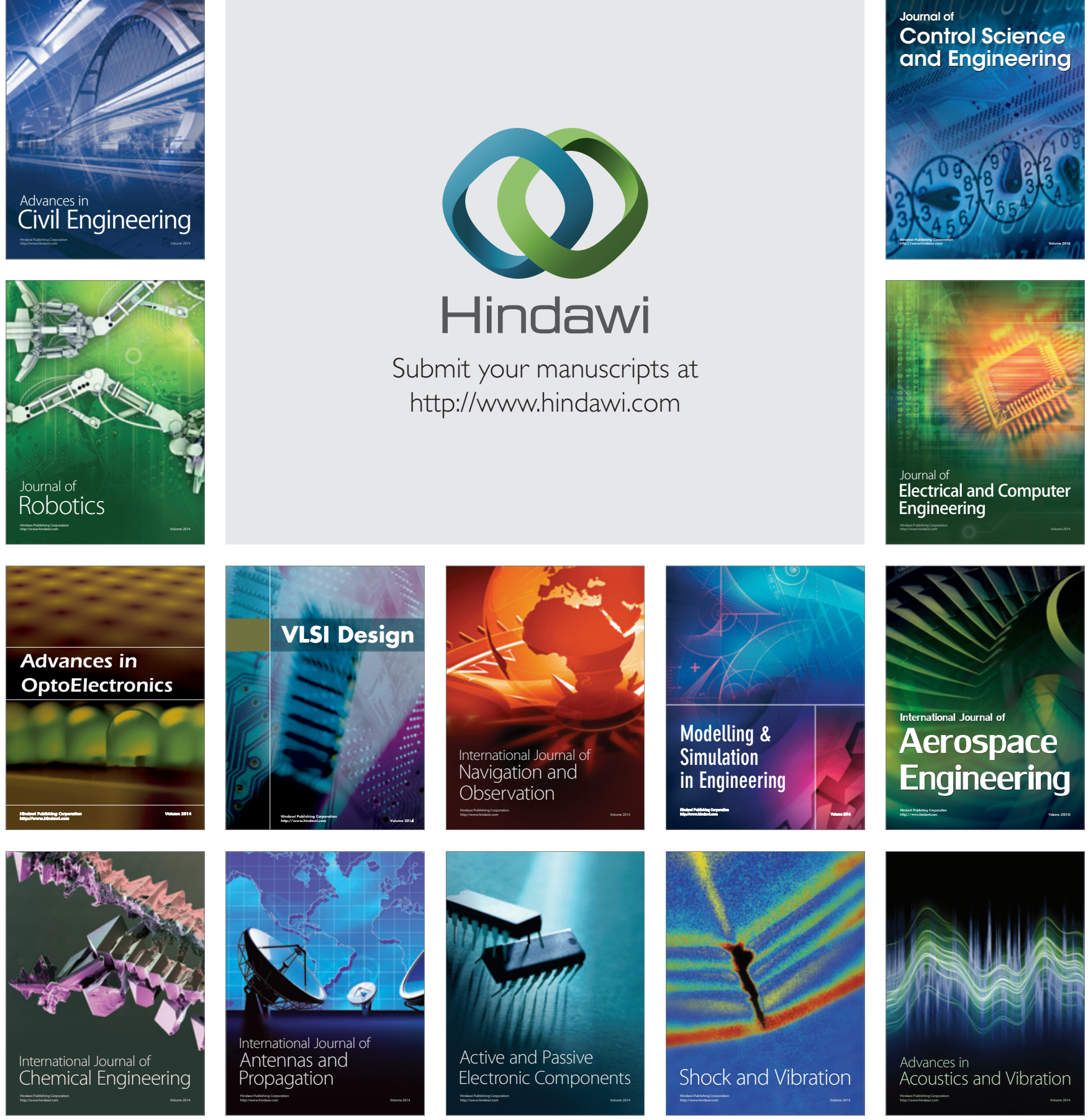